\title{
Two-scale expansion with drift approach to the Taylor dispersion for reactive transport through porous media
}

\author{
Grégoire Allaire $^{\mathrm{a}, 1,2,3}$, Robert Brizzi ${ }^{\mathrm{a}, 1}$, Andro Mikelić $^{*, \mathrm{~b}, 1}$, Andrey Piatnitski ${ }^{\mathrm{c}}$ \\ ${ }^{a}$ CMAP, Ecole Polytechnique, F-91128 Palaiseau, France \\ ${ }^{b}$ Université de Lyon, Lyon, F-69003, France; Université Lyon 1, Institut Camille Jordan, UMR 5208, UFR Mathématiques, 43, \\ Bd du 11 novembre 1918, 69622 Villeurbanne Cedex, France \\ ${ }^{c}$ Narvik University College, Norway and Lebedev Physical Institute, Moscow, Russia
}

\begin{abstract}
In this work we study reactive flows through porous media. We suppose dominant Peclet's number, dominant Damköhler's number and general linear reactions at the pore boundaries. Our goal is to obtain the dispersion tensor and the upscaled model. We introduce the multiple scale expansions with drift for the problem and use this technique to upscale the reactive flow equations. Our result is illustrated with numerical simulations for the dispersion tensor.
\end{abstract}

Key words:

\section{Introduction}

Our understanding of porous media flows comes from the knowledge of basic physical principles at the pore level and from observations at the macroscale. Solving multiphase multicomponent Navier-Stokes equations at the level of pores (the fine scale) requires gathering of tremendous amount of fine scale data. Consequently, the present computational resources are not able to handle such flows. Furthermore, it is almost impossible to obtain a complete description of the geometry and of the ongoing chemical process.

To circumvent this difficulty a usual approach is to describe the essential physical behavior in an averaged sense. This corresponds to upscaling from the

\footnotetext{
${ }^{*}$ Corresponding author

Email addresses: gregoire.allaire@polytechnique.fr (Grégoire Allaire), robert.brizzi@polytechnique.fr (Robert Brizzi), mikelic@univ-lyon1.fr (Andro Mikelić), andrey@sci.lebedev.ru (Andrey Piatnitski)

${ }^{1}$ The research was partially supported by the GNR MOMAS CNRS-2439 (Modélisation Mathématique et Simulations numériques liées aux problèmes de gestion des déchets nucléaires) (PACEN/CNRS, ANDRA, BRGM, CEA, EDF, IRSN).

${ }^{2} \mathrm{G}$. A. is a member of the DEFI project at INRIA Saclay Ile-de-France.

${ }^{3}$ G.A. is partially supported by the Chair "Mathematical modelling and numerical simulation, F-EADS - Ecole Polytechnique - INRIA"
}

microscale to the macroscale, where we do not have to consider all finer scale details.

There are different approaches to the upscaling of flows through porous media. Early references involve the method of moments (Aris, Brenner), more recent papers use either volume averaging or multiple scale expansions. The multiple scale expansions have the advantage of making the results mathematically rigorous by means of the homogenization method.

The simplest flow type in porous media is single phase single component flow. Here upscaling of the Navier-Stokes equations gives Darcy's law. The multiple scale expansion was constructed by Ene and Sanchez-Palencia and the approximation was justified by Tartar using the homogenization method. For a review of the classic results on derivation of Darcy's law, with detailed references, we refer to [2].

Next important question linked with the saturated flow through porous media is the upscaling of tracer dispersion. The transverse diffusion causes the particle cloud, which is transported by the flow, to undergo a transition from the pore level convectiondiffusion to a convection-dispersion phenomenon at the macroscale. The observed spreading is called hydrodynamic dispersion. Its effects are closely linked with the size of the Péclet number and for diffusive transport through porous media we are typically in Taylor dispersion-mediated mixing, which means that 
we have a dominant Péclet number smaller than a threshold value. When Péclet's number reaches that threshold value, then diffusive transport changes its behavior to turbulent mixing.

The theoretical study of the dispersion goes back to the pioneering paper of Taylor [39], where an explicit expression for effective dispersion in cylindrical capillaries was found. It led to thousands of articles on dispersion in capillaries. It is interesting to note that recent mathematical analysis from [29] and [13] showed that Taylor's dispersion theory is valid for all Péclet's numbers smaller than the threshold value, corresponding to the characteristic small scale diffusion and the characteristic global advection times of same order. This was advocated through numerous numerical experiments in [16], where Péclet's numbers corresponding to Taylor's experiments from [39] were determined and found to be close to the threshold value.

For study of dispersion in porous media using an averaging technique, we refer to [12] and [34]. The upscaled system is obtained by making the ad hoc closure hypothesis that the perturbation of the upscaled concentration is proportional to its gradient.

Study of the dispersion in porous media via analysis by multiple scale expansions was undertaken in a number of papers. Papers [36] and [27] focused at the important case when Péclet number is of order $\varepsilon^{-1}$, where $\varepsilon$ is the characteristic pore size. In [8] dispersion was studied for various magnitudes of Péclet's numbers. The systematic study of the dispersion tensor is in [37] and in [7].

Presence of the chemical reactions complicates the situation further. Already for reactive flows through capillaries the literature is reduced to several recent papers (see [16] for references). We mention modeling dispersion for a flow in a biporous media with adsorption in the micropores, in [11] using multiple scale expansions technique from [9]. Multiple scale expansion for reactive flows with dominant Péclet's number and with infinite rate constant for adsorption is in [24]. The case of infinite linear adsorption constant is considered in [26].

In most situations it turned out that the multiple scale expansions could be done rigourously using a particular homogenization tool called the twoscale convergence. It was introduced by Nguentseng and Allaire and we refer to [1] for a complete theory with applications and references. It was generalized to cover also presence of surface terms in [4] and
[30]. The two-scale convergence not only justifies using multiple scales expansions but also for very complex structures is simpler than expansions, because it necessitates less computations. An example is obtaining Biot's equations from poroelasticity (for review see [28]). With such motivation, Piatnitski et al introduced in [14] and [25] the two-scale convergence with drift. See also [3] for a detailed theory. Then it was applied with success in [5] to reactive flows with volume reactions and infinite linear adsorption const! ant at pore boundaries.

In this paper we present for the first time in the engineering literature the multiple scale expansion with drift applied to reactive flows through porous media with dominant Péclet's and Damköhler's numbers and general linear surface reactions. It was anticipated in [31], pages 212-216, and is closely linked with theoretical notion of the two-scale convergence with drift. The question of the rigorous mathematical justification of the upscaling is addressed in the preprint [6]. In this article we present the model, apply the multiple scale expansion with drift to it and obtain formally the upscaled model. Then it is illustrated with numerical simulations for the dispersion matrix. We note that in [8] and [37] it was necessary to use two time scales in order to get the correct result. Here we will see that the approach is more elegant and calculations shorter.

\section{Examples of reactive flows}

We consider reactive transport of solute particles transported by a stationary incompressible viscous flow through a porous medium. The flow regime is assumed to be laminar through the fluid part $\Omega_{f}$ of this porous medium, which is supposed to be a network of interconnected channels. The flow satisfies a slip (non penetrating) condition on the fluid/solid interfaces and $\Omega_{f}$ is saturated by the fluid. Solute particles are participants in a chemical reaction with the solid boundaries of the pores.

\subsection{Model for reactive transport of a single solute}

This is the simplest example and it is described by the following model for the solute concentration $c^{*}$ and for the surface concentration $\hat{c}^{*}$ :

$$
\frac{\partial c^{*}}{\partial t^{*}}+\mathbf{v}^{*}\left(x^{*}, t^{*}\right) \cdot \nabla_{x^{*}} c^{*}-D^{*} \nabla_{x^{*}}^{2} c^{*}=0 \text { in } \Omega_{f},
$$

where $\mathbf{v}^{*}$ is the given fluid velocity (obtained e.g. by solving the Navier-Stokes equations), and $D^{*}$ the 
molecular diffusion (a positive constant). At the solidfluid boundary $\partial \Omega_{f}$ takes place an assumed linear adsorption process, described by the following equations:

$$
-D^{*} \nabla_{x^{*}} c^{*} \cdot \mathbf{n}=\frac{\partial \hat{c}^{*}}{\partial t^{*}}=\hat{k}^{*}\left(c^{*}-\frac{\hat{c}^{*}}{K^{*}}\right) \quad \text { on } \partial \Omega_{f},
$$

where $\hat{k}^{*}$ represents the rate constant for adsorption, $K^{*}$ the linear adsorption equilibrium constant and $\mathbf{n}$ is the unit normal at $\partial \Omega_{f}$ oriented outwards with respect to $\Omega_{f}$.

\subsection{Model for the binary ion exchange}

We now consider another, more complex model, namely ion exchange with two species. The binding on the pore surfaces is due to electric charges carried by the solutes and the exchange complex. For a detailed mathematical modeling and references from the chemical engineering we refer to [15]. Let us just briefly recall the equations.

For $i=1,2$, let $M_{i}$ denote the ion $i$ in solution, let $\bar{M}_{i}$ denote the ion $i$ attached to the exchange complex and let $n_{i}$ denote the valence of ion $i$. In order to maintain electroneutrality the exchange reaction has the form

$$
n_{2} M_{1}+n_{1} \bar{M}_{2} \rightleftarrows n_{1} M_{2}+n_{2} \bar{M}_{1} .
$$

The reaction rate from left to the right will be denoted by $\tilde{k}_{1}$, and from right to left by $\tilde{k}_{2}$. In models for the binary ion exchange differences in molecular diffusivities are neglected. Hence in $\Omega_{f}$ we have equation (1) for both concentrations $c_{i}^{*}, i=1,2$. At the solid/fluid boundaries $\partial \Omega_{f} \backslash \partial \Omega$ we suppose the following rate description for the adsorption reaction:

$$
\frac{\partial s_{i}^{*}}{\partial t^{*}}=F_{i}\left(c_{1}^{*}, s_{1}^{*}, c_{2}^{*}, s_{2}^{*}\right), \quad i=1,2,
$$

where $F_{1}$ and $F_{2}$ are given by $F_{1}=n_{2}\left(\tilde{k}_{1}-\tilde{k}_{2}\right)$, $F_{2}=n_{1}\left(\tilde{k}_{2}-\tilde{k}_{1}\right)$ and $c_{i}^{*}$ and $s_{i}^{*}$ denote the concentrations of $M_{i}$ and $\bar{M}_{i}$, respectively. Valences $n_{i}, i=1,2$ are supposed to be bigger than or equal to 1 .

In the engineering literature it is observed that the ion exchange capacity $\bar{s}^{*}=n_{1} s_{1}^{*}+n_{2} s_{2}^{*}$ is conserved. Following [15] and [23], we find out that $\left\{\bar{c}^{*}, \bar{s}^{*}\right\}$, with $\bar{c}^{*}=n_{1} c_{1}^{*}+n_{2} c_{2}^{*}$, satisfies the equations

$$
\frac{\partial \bar{c}^{*}}{\partial t^{*}}+\mathbf{v}^{*}\left(x^{*}, t^{*}\right) \nabla_{x^{*}} \bar{c}^{*}-D^{*} \nabla_{x^{*}}^{2} \bar{c}^{*}=0 \text { in } \Omega_{f} \times(0, T),
$$

$$
-D^{*} \nabla_{x^{*}} \bar{c}^{*} \cdot \mathbf{n}=\frac{\partial \bar{s}}{\partial t^{*}}=0 \quad \text { on } \partial \Omega_{f} \backslash \partial \Omega .
$$

Hence $\bar{s}^{*}=\left.n_{1} s_{1}^{*}\right|_{t=0}+\left.n_{2} s_{2}^{*}\right|_{t=0}$. Next we observe that

$$
s_{2}^{*}=\frac{1}{n_{2}}\left(\bar{s}^{*}-n_{1} s_{1}^{*}\right) ; \quad c_{2}^{*}=\frac{1}{n_{2}}\left(\bar{c}^{*}-n_{1} c_{1}^{*}\right)
$$

and it is enough to study the corresponding problem for $\left\{c_{1}^{*}, s_{1}^{*}\right\}$.

We note that the system (4)-(5) does not contain chemical reactions. Hence $\left\{\bar{c}^{*}, \bar{s}^{*}\right\}$ are calculated independently and then we turn to the determination of $c_{i}^{*}$ and $s_{i}^{*}, i=1,2$.

Following [15] the reaction rates appearing in (3) are

$$
\left\{\begin{array}{l}
\tilde{k}_{1}=k_{1}\left(c_{1}^{*}\right)^{n_{2}}\left(\frac{\bar{s}^{*}}{n_{1}}-s_{1}^{*}\right)^{n_{1}}, \quad \text { and } \\
\tilde{k}_{2}=k_{2}\left(s_{1}^{*}\right)^{n_{2}}\left(\frac{\bar{c}^{*}}{n_{1}}-c_{1}^{*}\right)^{n_{1}},
\end{array}\right.
$$

with $k_{1}=\hat{k}_{1} \gamma_{1}^{n_{2}} \delta_{2}^{n_{1}}\left(\frac{n_{1}}{n_{2}}\right)^{n_{1}}$ and $k_{2}=\hat{k}_{2} \gamma_{2}^{n_{1}} \delta_{1}^{n_{2}}\left(\frac{n_{1}}{n_{2}}\right)^{n_{1}}$ being positive constants. More general rate functions, corresponding to other chemical settings, are introduced similarly (see [15]). The isotherms (i.e. singular points) corresponding to the ordinary differential equation (ODE)

$$
\begin{gathered}
\frac{\partial s_{1}^{*}}{\partial t^{*}}=n_{2}\left(\tilde{k}_{1}-\tilde{k}_{2}\right)=n_{2}\left(k_{1}\left(c_{1}^{*}\right)^{n_{2}}\left(\frac{\bar{s}^{*}}{n_{1}}-s_{1}^{*}\right)^{n_{1}}-\right. \\
\left.k_{2}\left(s_{1}^{*}\right)^{n_{2}}\left(\frac{\bar{c}^{*}}{n_{1}}-c_{1}^{*}\right)^{n_{1}}\right)
\end{gathered}
$$

are studied in [15] where it was established that (8) defines a monotone isotherm.

In order to get isotherms we suppose that $\bar{c}^{*}$ does not depend on time and that initially it was a constant. Then $\bar{c}^{*}\left(x^{*}, t^{*}\right)=C_{0}^{*}=$ constant $>0$. For $\bar{s}^{*}$ we suppose $\bar{s}^{*}=\bar{s}_{0}^{*}=$ constant $>0$.

Let $\left\{c_{1}^{e q}, s_{1}^{e q}\right\}$ be such isotherm. Then

$$
\begin{gathered}
\frac{1}{n_{2}} \frac{\partial F_{1}}{\partial c_{1}^{*}}\left(c_{1}^{e q}, s_{1}^{e q}\right)=n_{2} k_{1}\left(c_{1}^{e q}\right)^{n_{2}-1}\left(\frac{\bar{s}^{*}}{n_{1}}-s_{1}^{e q}\right)^{n_{1}}+ \\
k_{2} n_{1}\left(s_{1}^{e q}\right)^{n_{2}}\left(\frac{\bar{c}^{*}}{n_{1}}-c_{1}^{e q}\right)^{n_{1}-1}=\frac{F_{1 c}}{n_{2}}>0 \\
\frac{1}{n_{2}} \frac{\partial F_{1}}{\partial s_{1}^{*}}\left(c_{1}^{e q}, s_{1}^{e q}\right)=-n_{1} k_{1}\left(c_{1}^{e q}\right)^{n_{2}}\left(\frac{\bar{s}^{*}}{n_{1}}-s_{1}^{e q}\right)^{n_{1}-1}- \\
k_{2} n_{2}\left(s_{1}^{e q}\right)^{n_{2}-1}\left(\frac{\bar{c}^{*}}{n_{1}}-c_{1}^{e q}\right)^{n_{1}}=-\frac{F_{1 s}}{n_{2}}<0
\end{gathered}
$$

and the linearized rate function reads

$$
\begin{gathered}
\frac{\partial s_{1}^{*}}{\partial t^{*}}=F_{1 c} c_{1}^{*}-F_{1 s} s_{1}^{*}=F_{1 c}\left(c_{1}^{*}-\frac{s_{1}^{*}}{\frac{F_{1 c}}{F_{1 s}}}\right) \\
=-D^{*} \nabla_{x^{*}} c_{1}^{*} \cdot \mathbf{n} .
\end{gathered}
$$




\subsection{Reactive flow systems with $m$ species}

We investigate again another model of ion exchange reactions, or more general adsorption reactions, between an aqueous solution involving mono and multi-valent cations and $N_{G}$ different exchange sites. It is based on the following set of simultaneous chemical reactions

$$
\begin{gathered}
n_{j} M_{i}+n_{i} M_{j}\left(E_{\alpha}\right)_{n_{j}} \rightleftarrows n_{i} M_{j}+n_{j} M_{i}\left(E_{\alpha}\right)_{n_{i}}, \\
\left(\alpha=1, \ldots, N_{G}\right), i, j=1, \ldots, m,
\end{gathered}
$$

where $M_{i}$ designates the i-th cation with valence $n_{i}$ and $E_{\alpha}$ signifies an exchange site of type $\alpha$ with unit charge.

We are interested in the pore level modeling of the reactive flows of $m$ solutes. The general mathematical reference is the book [19]. Chemistry is presented there through non-linear source terms and the approach is very general. In this paper we would like to apply our approach to particular reactive flows with adsorption at the pore boundaries. A mathematically oriented reference with detailed modeling is the book [35]. In fact they present models with the surface terms already scaled up to volume ones. Also the molecular diffusion is neglected. Nevertheless, the adsorption modeling is given in details.

We start by recalling the basic quantitative description of the adsorption mechanism. If there is a finite rate of transfer from solution to adsorbent, then one assumes that this rate depends on the pertinent parameters of the problem. Frequently, it is assumed that the rate of transfer of solute is determined by the rate of transfer of mass through the stagnant film about the particle, other processes occurring at equilibrium. Let $s_{i}^{*}$ be the concentration of the i-th solute on the solid in moles per unit surface. Then we have

$$
\frac{\partial s_{i}^{*}}{\partial t^{*}}=k\left(c_{i}^{*}-c_{i}^{* *}\right),
$$

where $k$ is the rate velocity and $c_{i}^{* *}$ the concentration in the fluid if it was in equilibrium with the adsorbed phase. $c_{i}^{* *}$ are given non-linear functions of $s_{i}^{*}$, corresponding to the isotherms.

If a local equilibrium were established everywhere at any time, then the surface concentrations $s_{i}^{*}$ would be related to the volume concentrations $c_{i}^{*}$ by

$$
s_{i}^{*}=f_{i}\left(c_{1}^{*}, \ldots, c_{m}^{*}\right), \quad i=1, \ldots, m .
$$

Typical example is the Langmuir adsorption isotherm

$$
f_{i}\left(a_{1}, \ldots, a_{m}\right)=\frac{N_{i} K_{i} a_{i}}{1+\sum_{j=1}^{m} K_{j} a_{j}}, \quad i=1, \ldots, m,
$$

where $K_{i}=k_{1 i} / k_{2 i}$ is the ratio of the rate velocities (of adsorption and desorption) and $N_{i}$ is the limiting value of $s_{i}^{*}$ (the maximum number of moles of solute $i$ that can be adsorbed per unit of adsorbent).

Nonlinear mappings $f_{i}$ define a diffeomorphism on a subset of $\mathbb{R}^{m}$ and we have

$$
c_{i}^{* *}=\frac{s_{i}^{*}}{K_{i} N_{i}} \frac{1}{1-\sum_{j=1}^{m} s_{j}^{*} / N_{j}}, \quad i=1, \ldots, m .
$$

Properties of the Jacobian matrix $\left[\frac{\partial f_{i}}{\partial c_{j}^{*}}\right]$ are discussed in [35], Vol. 2, chapter 3, in the context of the study of the generalized Riemann invariants. It is proved that the matrix $\left[\frac{\partial f_{i}}{\partial c_{j}^{*}}\right]$ has $m$ real distinct eigenvalues. It is important to note that this matrix is neither symmetric nor normal and it is diagonalized only by a similarity transform, which is not orthogonal.

We are interested in a linearized problem. We suppose that there is a constant equilibrium state $\left(c_{1 e}, \ldots, c_{m e}\right) ; \quad s_{i e}=f_{i}\left(c_{1 e}, \ldots, c_{m e}\right)$. This is a positive steady solution for the ODE on the surface. Then we study the perturbation of that solution

$$
c_{1 e}+c_{1}^{*}, \ldots, c_{m e}+c_{m}^{*} ; \quad s_{1 e}+s_{1}^{*}, \ldots, s_{m e}+s_{m}^{*}
$$

Then, using that $c_{i e}-c_{i}^{* *}\left(s_{1 e}, \ldots, s_{m e}\right)=0$, we have

$$
\begin{gathered}
c_{i e}+c_{i}^{*}-c_{i}^{* *}\left(s_{1 e}+s_{1}^{*}, \ldots, s_{m e}+s_{m}^{*}\right)=c_{i}^{*}- \\
\left.\sum_{j=1}^{m}\left[\frac{\partial c_{i}^{* *}}{\partial s_{j}^{*}}\right]\right|_{s^{*}=s_{e}} s_{j}^{*}+\mathcal{O}\left(\left|s^{*}\right|^{2}\right) .
\end{gathered}
$$

Hence the linearized law (12) reads

$\frac{\partial s_{i}^{*}}{\partial t^{*}}=k\left(c_{i}^{*}-\sum_{j=1}^{m} F_{i j} s_{j}^{*}\right)=-D^{*} \nabla c_{i}^{*} \cdot \mathbf{n}, \quad i=1, \ldots, m$,

where

$$
F_{i j}= \begin{cases}\frac{c_{i e}}{N_{j}}\left(1+\sum_{j=1}^{m} K_{j} c_{j e}\right), & j \neq i \\ \frac{1}{N_{i}}\left(\frac{1}{K_{i}}+c_{i e}\right)\left(1+\sum_{j=1}^{m} K_{j} c_{j e}\right), & j=i .\end{cases}
$$


Since the application defining Langmuir isotherm is bijective, we know that $F$ is invertible. Nevertheless, $F$ is not symmetric. Following [35], Vol. 2, chapter 3 , we know that $F$ has $m$ distinct and real eigenvalues and there is a regular matrix $S$ such that

$$
S F S^{-1}=\Lambda=\operatorname{diag}\left(\lambda_{1}, \ldots, \lambda_{m}\right) .
$$

Our microscopic system for $\mathbf{c}^{*}=\operatorname{diag}\left(c_{1}^{*}, \ldots, c_{m}^{*}\right)$ and $\mathbf{s}^{*}=\operatorname{diag}\left(s_{1}^{*}, \ldots, s_{m}^{*}\right)$ is

$$
\begin{gathered}
\frac{\partial \mathbf{c}^{*}}{\partial t^{*}}+\left(\mathbf{v}^{*} \cdot \nabla_{x^{*}}\right) \mathbf{c}^{*}=D^{*} \nabla_{x^{*}}^{2} \mathbf{c}^{*} \quad \text { in } \Omega_{f} \\
\mathbf{c}^{*}\left(x^{*}, 0\right)=\mathbf{c}_{0}^{*}\left(x^{*}\right), \quad x^{*} \in \Omega_{f}, \\
-D^{*} \nabla_{x^{*}} \mathbf{c}^{*} \cdot \mathbf{n}=\frac{\partial \mathbf{s}^{*}}{\partial t^{*}}=k^{*}\left(\mathbf{c}^{*}-F \mathbf{s}^{*}\right) \\
\text { on } \partial \Omega_{f} \backslash \partial \Omega \\
\mathbf{s}^{*}\left(x^{*}, 0\right)=\mathbf{s}_{0}^{*}\left(x^{*}\right), \quad x^{*} \in \partial \Omega_{f} .
\end{gathered}
$$

Now we apply the similarity transform $S$ to the problem (19)-(22) and obtain the following system for $\left\{S \mathbf{c}^{*}, S \mathbf{s}^{*}\right\}$ :

$$
\begin{gathered}
\frac{\partial S \mathbf{c}^{*}}{\partial t^{*}}+\left(\mathbf{v}^{*} \cdot \nabla_{x^{*}}\right)\left(S \mathbf{c}^{*}\right)=D^{*} \nabla_{x^{*}}^{2}\left(S \mathbf{c}^{*}\right) \quad \text { in } \Omega_{f} \\
S \mathbf{c}^{*}\left(x^{*}, 0\right)=S \mathbf{c}_{0}^{*}\left(x^{*}\right), \quad x^{*} \in \Omega_{f}, \\
-D^{*} \nabla_{x^{*}}\left(S \mathbf{c}^{*}\right) \cdot \mathbf{n}=\frac{\partial S \mathbf{s}^{*}}{\partial t^{*}}=k^{*}\left(S \mathbf{c}^{*}-\Lambda S \mathbf{s}^{*}\right) \\
\text { on } \partial \Omega_{f} \backslash \partial \Omega \\
S \mathbf{s}^{*}\left(x^{*}, 0\right)=S \mathbf{s}_{0}^{*}\left(x^{*}\right), \quad x^{*} \in \partial \Omega_{f} .
\end{gathered}
$$

Again we are back to the homogenization problem (29)-(32), but this time for every component of $S \mathbf{c}^{*}$.

\section{Dimensionless equations and the assump- tions on the data}

The main conclusion of the previous section is that after linearization we are back to system (1)(2) and that it should be the starting point for the upscaling procedure.

To make an asymptotic analysis of the problem we must first introduce appropriate scales deduced from characteristic parameters. We denote them by a $R$-index (meaning "reference"). The characteristic length $L_{R}$ coincides in fact with the "observation distance". We assume that the typical heterogeneities in $\Omega_{f}$ have a characteristic size $\ell<<L_{R}$. We set $\varepsilon=\frac{\ell}{L_{R}}<<1$ and the rescaled flow domain is now

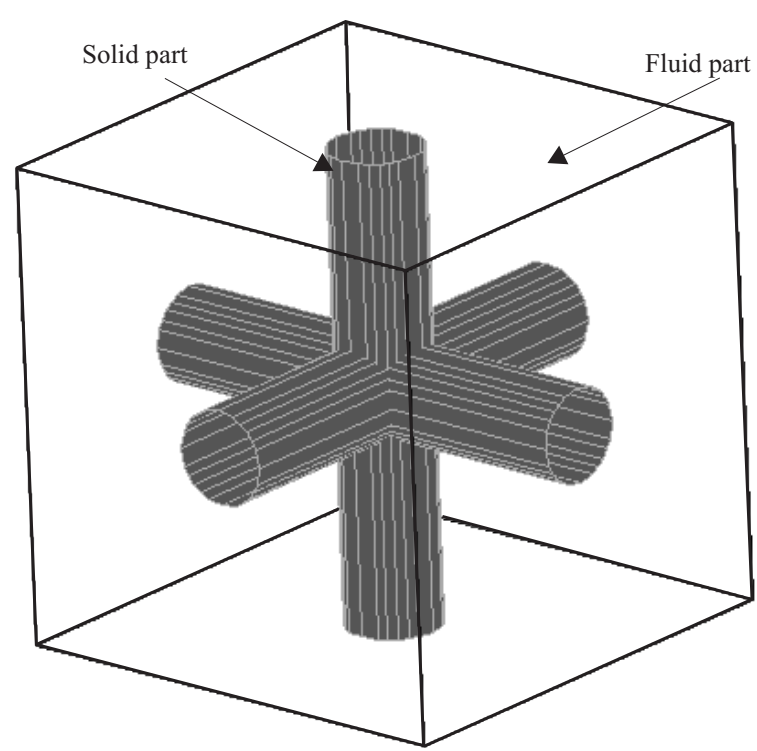

Figure 1: The unit pore $Y$ with the solid part $\Sigma^{0}$ (dark grey) and the remaining fluid part $Y^{0}=Y \backslash \Sigma^{0}$.

$\Omega_{\varepsilon}=\Omega_{f} / L_{R}$, with notation reminding us that it contains pores of characteristic non-dimensional size $\varepsilon$. Setting

$$
\begin{aligned}
& u_{f}=\frac{c^{*}}{c_{R}}, x=\frac{x^{*}}{L_{R}}, t=\frac{t^{*}}{T_{R}}, \mathbf{v}(x, t)=\frac{1}{V_{R}} \mathbf{v}^{*}\left(x^{*}, t^{*}\right), \\
& v_{s}=\frac{\hat{c}^{*}}{\hat{c}_{R}}, \text { we obtain the dimensionless equations } \\
& \frac{\partial u_{f}}{\partial t}+\frac{V_{R} T_{R}}{L_{R}} \mathbf{v}(x, t) \cdot \nabla_{x} u_{f}-\frac{D^{*} T_{R}}{L_{R}^{2}} \nabla_{x}^{2} u_{f}=0 \text { in } \Omega_{\varepsilon} .
\end{aligned}
$$

and

$$
-\frac{D^{*}}{L_{R}} c_{R} \nabla_{x} u_{f} \cdot \mathbf{n}=\frac{\hat{c}_{R}}{T_{R}} \frac{\partial v_{s}}{\partial t}=\hat{k}^{*}\left(c_{R} u_{f}-\frac{\hat{c}_{R} v_{s}}{K^{*}}\right) \text { on } \partial \Omega_{\varepsilon} .
$$

This problem involves the following time scales:

$T_{L}=$ characteristic global advection time $=\frac{L_{R}}{V_{R}}$

$T_{D}=$ characteristic global diffusion time $=\frac{L_{R}^{2}}{D^{*}}$

$T_{D E}=\frac{K^{*}}{\hat{k}^{*}}($ characteristic desorption time $)$

$T_{A}=\frac{\hat{c}_{R}}{c_{R} \hat{k}^{*}}$ (characteristic adsorption time)

$T_{\text {react }}=$ superficial chemical reaction time $=\frac{L_{R}}{\hat{k}^{*}}$ 
and the following characteristic non-dimensional numbers

$\mathbf{P e}=\frac{L_{R} V_{R}}{D^{*}}=\frac{T_{D}}{T_{L}}=\frac{\mathbf{P e}_{l o c}}{\varepsilon}$ (Péclet's number); $\mathbf{D a}=\frac{L_{R} \hat{k}^{*}}{D^{*}}=\frac{T_{D}}{T_{\text {react }}}=\frac{\mathbf{D a}_{l o c}}{\varepsilon}$ (Damköhler's num-

We choose to study a regime for which $T_{R}=T_{D}$, i.e. we are interested at dispersion at global diffusion time.

Let us be a little more precise on the definition of $\Omega_{\varepsilon}$. From now on we assume that $\Omega_{\varepsilon}$ is an $\varepsilon$-periodic open subset of $\mathbb{R}^{n}, n=2,3$. The unit periodicity cell is $Y=(0,1)^{n}$ on which we consider a smooth partition $\Sigma^{0} \cup Y^{0}$ where $\Sigma^{0}$ is the solid part and $Y^{0}$ is the fluid part (its periodic extension is a smooth connected open subset) (see Fig. 1). By periodic translations we obtain $Y_{\varepsilon}^{j}=\varepsilon\left(Y^{0}+j\right), \Sigma_{\varepsilon}^{j}=\varepsilon\left(\Sigma^{0}+\right.$ $j), S_{\varepsilon}^{j}=\varepsilon\left(\partial \Sigma^{0}+j\right), \Omega_{\varepsilon}=\bigcup_{j \in \mathbb{Z}^{n}} Y_{\varepsilon}^{j}$ and $S_{\varepsilon}=\partial \Omega_{\varepsilon}$. $\Omega_{\varepsilon}$ is supposed to be connected and its boundary $S_{\varepsilon}$ smooth.

The equations for $u_{\varepsilon}=u_{f}$ and $v_{\varepsilon}=v_{s}$ in their non-dimensional form read (with the velocity $\mathbf{v}_{\varepsilon}=\mathbf{v}$ )

$$
\begin{gathered}
\frac{\partial u_{\varepsilon}}{\partial t}+\mathbf{P e} \mathbf{v}_{\varepsilon}(x, t) \cdot \nabla_{x} u_{\varepsilon}=\nabla_{x}^{2} u_{\varepsilon} \text { in } \Omega_{\varepsilon} \times(0, T) \\
u_{\varepsilon}(x, 0)=u^{0}(x), \quad x \in \Omega_{\varepsilon} \\
-\nabla_{x} u_{\varepsilon} \cdot \mathbf{n}=\frac{T_{A}}{T_{\text {react }}} \frac{\partial v_{\varepsilon}}{\partial t} \\
=\frac{T_{D}}{T_{\text {react }}}\left(u_{\varepsilon}-\frac{T_{A}}{T_{D E}} v_{\varepsilon}\right) \text { on } \partial \Omega_{\varepsilon} \times(0, T) \\
v_{\varepsilon}(x, 0)=v^{0}(x), \quad x \in \partial \Omega_{\varepsilon}
\end{gathered}
$$

In the present work we make the following two hypotheses.

\section{HYPOTHESIS 1}

$\mathbf{P e} \sim \frac{1}{\varepsilon}, \mathbf{D a} \sim \frac{1}{\varepsilon}, \frac{T_{A}}{T_{\text {react }}}=\varepsilon r_{A r} \sim \varepsilon, \frac{T_{A}}{T_{D E}}=r_{A D} \sim 1$.

This hypothesis means that we choose to study the case with dominant Péclet and Damköhler numbers, with comparable characteristic adsorption and desorption times and longer reaction time. In particular it implies

$r_{A r} \sim 1, V_{R} \sim \hat{k}^{*}, D^{*} \sim \ell V_{R}, \hat{c}_{R} \sim \ell c_{R}$ and $K^{*} \sim \ell$.
The velocity field is periodic, i.e.

$$
\mathbf{v}_{\varepsilon}(x, t)=\mathbf{b}\left(\frac{x}{\varepsilon}\right)
$$

with a periodic divergence-free vector field $\mathbf{b}(y)$ satisfying

$$
\begin{gathered}
\max _{y \in \bar{Y}^{0}}|\mathbf{b}(y)| \leq C, \nabla_{y} \cdot \mathbf{b}(y)=0 \text { in } Y^{0}, \\
\mathbf{b}(y) \cdot \mathbf{n}(y)=0 \text { on } \partial \Sigma^{0} .
\end{gathered}
$$

Notice that the velocity could come from any type of incompressible flow.

The initial data are chosen such that $u^{0}(x) \in$ $L^{2}\left(\mathbb{R}^{n}\right)$ (i.e. $\left.\int_{\mathbb{R}^{n}}\left|u^{0}(x)\right|^{2} d x<+\infty\right)$ and $v^{0} \in H^{1}\left(\mathbb{R}^{n}\right)$ (i.e. $\left.\int_{\mathbb{R}^{n}}\left(\left|u^{0}(x)\right|^{2}+\left|\nabla u^{0}(x)\right|^{2}\right) d x<+\infty\right)$. Taking into account (33), we rewrite problem (29)-(32) as follows

$$
\begin{gathered}
\partial_{t} u_{\varepsilon}+\frac{\mathbf{P \mathbf { e } _ { l o c }}}{\varepsilon} \mathbf{b}\left(\frac{x}{\varepsilon}\right) \cdot \nabla u_{\varepsilon}-\nabla^{2} u_{\varepsilon}=0 \text { in } \Omega_{\varepsilon} \times(0, T), \\
-\frac{\partial u_{\varepsilon}}{\partial \mathbf{n}}=r_{A r} \partial_{t} v_{\varepsilon}=\frac{\mathbf{D a}_{l o c}}{\varepsilon}\left(u_{\varepsilon}-v_{\varepsilon} r_{A D}\right) \\
\text { on } \partial \Omega_{\varepsilon} \times(0, T), \\
u_{\varepsilon}(x, 0)=u^{0}(x), \quad v_{\varepsilon}(x, 0)=v^{0}(x),
\end{gathered}
$$

with

$$
r_{A r}=\varepsilon \frac{\hat{c}_{R}}{\ell c_{R}}
$$

where we recall that $\mathbf{P e}_{l o c}, \mathbf{D a} \mathbf{a}_{l o c}$ and $\frac{\hat{c}_{R}}{\ell c_{R}}$ are of order 1 with respect to $\varepsilon$.

Remark 1. If the velocity field $\mathbf{b}(y)$ is not divergencefree and/or does not satisfy the no-penetration condition $\mathbf{b}(y) \cdot \mathbf{n}(y)=0$ on $\partial \Sigma^{0}$, it is still possible to homogenize (34)-(36) by using first a factorization principle in the spirit of [5].

\section{Two-scale multiple expansion with drift}

We now introduce the method of two-scale asymptotic expansions with drift (see [31], [14] and [25]). More precisely, one assumes that

$$
u_{\varepsilon}(t, x)=\sum_{i=0}^{+\infty} \varepsilon^{i} u_{i}\left(t, x-\frac{\mathbf{b}^{*}}{\varepsilon} t, \frac{x}{\varepsilon}\right)
$$

\section{HYPOTHESIS 2}


with $u_{i}(t, x, y)$ a function of the macroscopic variable $x$ and of the periodic microscopic variable $y \in Y=$ $(0,1)^{n}$, and similarly

$$
v_{\varepsilon}(t, x)=\sum_{i=0}^{+\infty} \varepsilon^{i} v_{i}\left(t, x-\frac{\mathbf{b}^{*}}{\varepsilon} t, \frac{x}{\varepsilon}\right)
$$

Note that the effective drift velocity $\mathbf{b}^{*}$ is an unknown constant vector and its determination is part of the problem.

In the absence of convection and chemical reactions, it is known that the above two-scale expansion, with $\mathbf{b}^{*}=0$, gives the correct upscaled problem (see e.g. the classical textbooks [10] and [38]). Otherwise the classical approach (i.e. with $\mathbf{b}^{*}=0$ ) leads to a contradiction when solving the equations at the level of the representative elementary volume (REV), see below the so-called Fredholm alternative. Auriault and Adler avoided this difficulty in [8] by introducing an additional, artificial, time scale. Our novelty is to introduced an unknown drift velocity $\mathbf{b}^{*}$ which eliminated incompatibilities at the REV level, which is easy to handle in the calculations and which can be interpreted physically. We notice that the approach of Carbonell et al from [12], [32] and Quintard et al from [34] is based on using an ad hoc closure hypothesis, which we do not need.

Concerning the upscaling of reactive flows through porous media with small local Péclet number, the two-scale expansions were rigorously constructed in the references [22] and [21]. Notice that in this case the drift velocity is small and it is enough to apply our expansion with $\mathbf{b}^{*}=0$.

The idea is to plug this ansatz into equation (34). We note the corresponding chain rule differentiation:

$$
\begin{gathered}
\nabla\left[u_{\varepsilon}\left(t, x-\frac{\mathbf{b}^{*} t}{\varepsilon}, \frac{x}{\varepsilon}\right)\right]=\left(\varepsilon^{-1} \nabla_{y}+\nabla_{x}\right) u_{\varepsilon}\left(t, x-\frac{\mathbf{b}^{*} t}{\varepsilon}, \frac{x}{\varepsilon}\right), \\
\nabla^{2}\left[u_{\varepsilon}\left(t, x-\frac{\mathbf{b}^{*} t}{\varepsilon}, \frac{x}{\varepsilon}\right)\right]=\left(\varepsilon^{-2} \nabla_{y}^{2}+\frac{2}{\varepsilon} \operatorname{div}_{x} \nabla_{y}\right. \\
\left.+\nabla_{x}^{2}\right) u_{\varepsilon}\left(t, x-\frac{\mathbf{b}^{*}}{\varepsilon} t, \frac{x}{\varepsilon}\right),
\end{gathered}
$$

$\frac{\partial}{\partial t}\left[u_{\varepsilon}\left(t, x-\frac{\mathbf{b}^{*} t}{\varepsilon}, \frac{x}{\varepsilon}\right)\right]=\left(\frac{\partial}{\partial t}-\frac{\mathbf{b}^{*}}{\varepsilon} \nabla_{x}\right) u_{\varepsilon}\left(t, x-\frac{\mathbf{b}^{*} t}{\varepsilon}, \frac{x}{\varepsilon}\right)$.

After plugging all these expressions into the equations (34)-(36) and equating the coefficients in front of the same powers of $\varepsilon$, we get the following cascade of equations:

\section{Equation of order $\varepsilon^{-2}$ :}

$$
\begin{cases}\mathbf{P e}_{l o c} \mathbf{b}(y) \cdot \nabla_{y} u_{0}-\nabla_{y}^{2} u_{0}=0 & \text { in } Y^{0} \\ -\nabla_{y} u_{0} \cdot \mathbf{n}=0=u_{0}-v_{0} r_{A D} & \text { on } \partial \Sigma^{0} \\ \left\{u_{0}, v_{0}\right\} \quad \text { is } Y^{0} \text {-periodic. } & \end{cases}
$$

We deduce

$u_{0}(t, x, y) \equiv u_{0}(t, x)$ and $v_{0}(t, x, y) \equiv \frac{u_{0}(t, x)}{r_{A D}}$.

$$
\begin{aligned}
& \text { Equation of order } \varepsilon^{-1}: \\
& \left\{\begin{array}{l}
\mathbf{P e}_{l o c} \mathbf{b}(y) \cdot \nabla_{y} u_{1}-\nabla_{y}^{2} u_{1}=\left(\mathbf{b}^{*}-\right. \\
\left.\mathbf{P e}_{l o c} \mathbf{b}(y)\right) \cdot \nabla_{x} u_{0} \quad \text { in } Y^{0} \\
-\left(\nabla_{y} u_{1}+\nabla_{x} u_{0}\right) \cdot \mathbf{n}=-\frac{K^{*}}{\ell} \mathbf{b}^{*} \cdot \nabla_{x} u_{0} \\
=\mathbf{D a}_{l o c}\left(u_{1}-v_{1} r_{A D}\right) \quad \text { on } \partial \Sigma^{0} ; \\
\left\{u_{1}, v_{1}\right\} \quad \text { is } Y^{0} \text {-periodic. }
\end{array}\right.
\end{aligned}
$$

We deduce

$$
v_{1}=\frac{1}{r_{A D}}\left(u_{1}+\frac{D^{*} K^{*}}{\hat{k}^{*} \ell^{2}} \mathbf{b}^{*} \cdot \nabla_{x} u_{0}\right)
$$

and we search for $u_{1}(t, x, y)$ of the form

$$
u_{1}(t, x, y)=\sum_{i=1}^{n} \frac{\partial u_{0}}{\partial x_{i}}(t, x) w_{i}(y) .
$$

For $w_{i}$ we have a cell problem which reads as follows:

$$
\left\{\begin{array}{l}
\mathbf{P e}_{l o c} \mathbf{b}(y) \cdot \nabla_{y} w_{i}-\nabla_{y}^{2} w_{i}= \\
b_{i}^{*}-\mathbf{P e}_{l o c} b_{i}(y) \\
-\left(\nabla_{y} w_{i}+\mathbf{e}^{i}\right) \cdot \mathbf{n}=-\frac{K^{*}}{\ell} b_{i}^{*} \text { on } \partial \Sigma^{0} \\
w_{i} \quad \text { is } Y^{0}-\text { periodic. }
\end{array}\right.
$$

It has the same differential operator as in problems (37) and (39): a feature of this operator is that it is not invertible. Consequently, in order to solve such problems we need an appropriate tool which is the following Fredholm alternative.

Fredholm alternative for the cell problem: the boundary value problem

$$
\left\{\begin{array}{l}
\mathbf{P e}_{l o c} \mathbf{b}(y) \cdot \nabla_{y} \zeta(y)-\nabla_{y}^{2} \zeta(y)=g(y) \text { in } Y^{0} \\
-\nabla_{y} \zeta(y) \cdot \mathbf{n}=h(y) \text { on } \partial \Sigma^{0} \\
\zeta \quad \text { is } Y^{0}-\text { periodic }
\end{array}\right.
$$

has a unique solution in $H^{1}\left(Y^{0}\right)$, up to an additive constant, if and only if the compatibility condition

$$
\int_{Y^{0}} g(y) d y-\int_{\partial \Sigma^{0}} h(y) d S=0 .
$$


is satisfied. Applying the Fredholm alternative to (41), i.e. requiring the compatibility condition (43) to hold true, yields the value of the drift $\mathbf{b}^{*}$ :

$$
\mathbf{b}^{*}=\frac{\mathbf{P e}_{l o c}}{\left|Y^{0}\right|+\left|\partial \Sigma^{0}\right|_{n-1} \frac{K^{*}}{\ell}} \int_{Y^{0}} \mathbf{b}(y) d y=\mathbf{P e}_{l o c} \overline{\mathbf{b}}
$$

where $\left|Y^{0}\right|$ is the relative volume of the fluid part of the unit cell (the porosity) and $\left|\partial \Sigma^{0}\right|_{n-1}$ is the dimensionless pore surface area in the unit cell (the tortuosity). Formula (44) ensures that $w_{i}$ exists and is unique up to an additive constant. Multiplying (41) by $w_{i}$ and integrating by parts yields the equality

$$
\begin{gathered}
\int_{Y^{0}}\left|\nabla_{y} w_{i}\right|^{2} d y=\mathbf{P e}_{l o c} \int_{Y^{0}}\left(\bar{b}_{i}-b_{i}\right) w_{i} d y+ \\
\int_{\partial \Sigma^{0}}\left(\frac{K^{*}}{\ell} \mathbf{P} \mathbf{e}_{l o c} \bar{b}_{i}-\mathbf{e}^{i} \cdot \mathbf{n}\right) w_{i} d S_{y},
\end{gathered}
$$

from which we deduce that $\mathbf{w}$ has at most linear growth in $\mathbf{P} \mathbf{e}_{l o c}$.

Equation of order $\varepsilon^{0}$ :

$\left\{\begin{array}{l}\mathbf{P e}_{l o c} \mathbf{b}(y) \cdot \nabla_{y} u_{2}-\nabla_{y}^{2} u_{2}=\nabla_{x}^{2} u_{0}-\partial_{t} u_{0}+ \\ \mathbf{P e}_{l o c}(\overline{\mathbf{b}}-\mathbf{b}(y)) \nabla_{x} u_{1}+2 \operatorname{div}_{x} \nabla_{y} u_{1} \text { in } Y^{0} \\ -\left(\nabla_{y} u_{2}+\nabla_{x} u_{1}\right) \cdot \mathbf{n}=\left(-\mathbf{P} \mathbf{e}_{l o c} \overline{\mathbf{b}} \cdot \nabla_{x} v_{1}\right. \\ \left.+\frac{\partial v_{0}}{\partial t}\right) \frac{\hat{c}_{R}}{\ell c_{R}}=\mathbf{D} \mathbf{a}_{l o c}\left(u_{2}-v_{2} r_{A D}\right) \text { on } \partial \Sigma^{0} \\ \left\{u_{2}, v_{2}\right\} \quad \text { is } Y^{0} \text { - periodic. }\end{array}\right.$

The compatibility condition for the existence of $u_{2}$ reads:

$$
\begin{gathered}
\int_{Y^{0}}\left(\mathbf{P e}_{l o c}(\overline{\mathbf{b}}-\mathbf{b}(y)) \cdot \nabla_{x} u_{1}+\right. \\
\left.\operatorname{div}_{x} \nabla_{y} u_{1}+\nabla_{x}^{2} u_{0}-\partial_{t} u_{0}\right) d y=\frac{\hat{c}_{R}}{\ell c_{R}} \int_{\partial \Sigma^{0}}\left(\frac{\partial v_{0}}{\partial t}\right. \\
\left.-\mathbf{P e}_{l o c} \overline{\mathbf{b}} \cdot \nabla_{x} v_{1}\right) d S_{y}=\frac{K^{*}}{\ell} \int_{\partial \Sigma^{0}}\left(\partial_{t} u_{0}-\operatorname{div}_{x}\{\right. \\
\left.\left.\mathbf{P e}_{l o c} \overline{\mathbf{b}} \otimes\left(\frac{K^{*} D^{*}}{\hat{k}^{*} \ell^{2}} \mathbf{P e}_{l o c} \overline{\mathbf{b}}+\mathbf{w}\right) \nabla_{x} u_{0}\right\}\right) d S_{y} .
\end{gathered}
$$

Let $K_{d}=\left|Y^{0}\right|+\frac{K^{*}}{\ell}\left|\partial \Sigma^{0}\right|_{n-1}$. After replacing $u_{1}$ by its previous value in terms of $\nabla_{x} u_{0}$ and $w_{i}$, we deduce from (46) the homogenized problem (47)-(48).

$$
\begin{gathered}
K_{d} \partial_{t} u_{0}=\operatorname{div}_{x}\left(A^{*} \nabla_{x} u_{0}\right) \text { in } \mathbb{R}^{n} \times(0, T), \\
u_{0}(x, 0)=\frac{\left|Y^{0}\right| u^{0}(x)+\frac{\hat{c}_{R}}{\ell c_{R}}\left|\partial \Sigma^{0}\right|_{n-1} v^{0}(x)}{\left|Y^{0}\right|+\frac{K^{*}}{\ell}\left|\partial \Sigma^{0}\right|_{n-1}} \text { in } \mathbb{R}^{n} .
\end{gathered}
$$

The effective diffusion tensor $A^{*}$ is defined merely by its inner product with the hessian matrix $\nabla^{2} u_{0}$, so that only its symmetric part contributes. It is defined by

$$
\begin{gathered}
A_{i j}^{*}=\int_{Y^{0}} \frac{\partial w_{j}}{\partial y_{i}} d y+\mathbf{P e}_{l o c}^{2} \frac{K^{*}}{\ell} \frac{K^{*} D^{*}}{\hat{k}^{*} \ell^{2}}\left|\partial \Sigma^{0}\right|_{n-1} \bar{b}_{i} \bar{b}_{j}- \\
\mathbf{P e}_{l o c} \int_{Y^{0}} w_{j}(y) b_{i}(y) d y+\mathbf{P e}_{l o c} \bar{b}_{i} \int_{Y^{0}} w_{j}(y) d y+ \\
\frac{K^{*}}{\ell} \mathbf{P e}_{l o c} \bar{b}_{i} \int_{\partial \Sigma^{0}} w_{j}(y) d S_{y}+\left|Y^{0}\right| \delta_{i j}
\end{gathered}
$$

which should be symmetrized. This is not the best form for studying the properties of the dispersion tensor $A^{*}$. After testing the equation (41) for $w_{i}$, by $w_{j}$ and using Green's formula and deleting the antisymmetric contribution, we get the following equivalent symmetric form of it:

$$
\begin{array}{r}
A^{*}=\mathbf{P e}_{l o c}^{2} \frac{K^{*}}{\ell} \frac{K^{*} D^{*}}{\hat{k}^{*} \ell^{2}}\left|\partial \Sigma^{0}\right|_{n-1} \overline{\mathbf{b}} \otimes \overline{\mathbf{b}} \\
+\int_{Y^{0}}\left(\mathbf{I}+\nabla_{y} \mathbf{w}(y)\right)\left(\mathbf{I}+\nabla_{y} \mathbf{w}(y)\right)^{\mathcal{T}} d y .
\end{array}
$$

Obviously the tensor $A^{*}$ is positive definite and problem (47)-(48) has a unique solution $u_{0}$. Furthermore $A^{*}$ has at most quadratic growth in $\mathbf{P e}_{l o c}$ (see [17], [20] for examples and counter-examples of such growth, depending on the geometry of the flow field $\mathbf{b}(y))$. It is clear from formula (50) that the dispersion tensor $A^{*}$ is usually not isotropic since $\overline{\mathbf{b}}$ is a privilegied direction. This fact is well recognized by making a difference between the longitudinal and transverse dispersion.

Remark 2. As suggested by the asymptotic expansion, the sequence $\left\{u_{\varepsilon}, v_{\varepsilon}\right\}$ of solutions to (34)-(36) satisfies

$$
\begin{array}{r}
u_{\varepsilon}(t, x)=u_{0}\left(t, x-\frac{\mathbf{P} \mathbf{e}_{l o c} \overline{\mathbf{b}}}{\varepsilon} t\right)+r_{\varepsilon}^{u}(t, x), \\
v_{\varepsilon}(t, x)=\frac{T_{D E}}{T_{A}} u_{0}\left(t, x-\frac{\mathbf{P \mathbf { e } _ { l o c }} \overline{\mathbf{b}}}{\varepsilon} t\right)+r_{\varepsilon}^{v}(t, x)
\end{array}
$$

with functions $r_{\varepsilon}^{u}$ and $r_{\varepsilon}^{v}$ vanishing as $\varepsilon \rightarrow 0$. This convergence result is proven rigorously in [6].

Remark 3. Convection is not seen in the homogenized equation (48) because the solution $u$ is defined in moving coordinates when compared to $u_{\varepsilon}$ and $v_{\varepsilon}$ in (51). However, (48) is equivalent to a convection diffusion equation by a simple change of reference frame. 
Indeed, introducing $\tilde{u}_{\varepsilon}(t, x)=u_{0}\left(t, x-\frac{\mathbf{P e}_{l o c} \overline{\mathbf{b}}}{\varepsilon} t\right)$, it is a solution of

$$
\left\{\begin{array}{c}
K_{d} \frac{\partial \tilde{u}_{\varepsilon}}{\partial t}+\mathbf{P e}_{l o c} \frac{\overline{\mathbf{b}}}{\varepsilon} \cdot \nabla \tilde{u}_{\varepsilon}-\operatorname{div}\left(A^{*} \nabla \tilde{u}_{\varepsilon}\right)=0 \\
\operatorname{in~} \mathbb{R}^{n} \times(0, T) \\
\tilde{u}_{\varepsilon}(0, x)=\frac{\left|Y^{0}\right| u^{0}(x)+\frac{\hat{c}_{R}}{\ell c_{R}}\left|\partial \Sigma_{0}\right|_{n-1} v^{0}(x)}{\left|Y^{0}\right|+\frac{K^{*}}{\ell}\left|\partial \Sigma_{0}\right|_{n-1}} \text { in } \mathbb{R}^{n}
\end{array}\right.
$$

\section{Numerical study of the effective dispersion tensor}

We now present some numerical tests in the twodimensional case obtained with the FreeFem ++ package [33]. For these simulations, Lagrange P1 finite elements, with a characteristic Galerkin upwinding, are used and the total number of vertices (or degrees of freedom) is 34077 (we checked that our results are converged under mesh refinement). The solid obstacles are isolated circular disks. We have the following data for the parameters.

\begin{tabular}{|l|l|}
\hline \hline PARAMETERS & VALUES \\
\hline Observation length $: L_{R}$ & $10 \mathrm{~m}$, \\
\hline Pore length $: \ell$ & $10^{-5} \mathrm{~m}$ \\
\hline$\varepsilon=\ell / L_{R}$ & $10^{-6}$ \\
\hline Characteristic velocity: $V_{R}:$ & $10^{-3} \mathrm{~m} / \mathrm{sec}$ \\
\hline Diffusion coefficient: $D^{*}:$ & $10^{-8} \mathrm{~m}^{2} / \mathrm{sec}$, \\
\hline Péclet's number: Pe : & $10^{6}$ \\
\hline Adsorption rate $\hat{k}^{*}:$ & $10^{-3} \mathrm{~m} / \mathrm{sec}$ \\
\hline Adsorption equilibrium constant $K^{*}$ & $10^{-5} \mathrm{~m}$ \\
\hline Damköhler's number: Da & $10^{6}$ \\
\hline Local Péclet's number: Pe & 1 \\
\hline Local Damköhler's number: Da & 1 \\
\hline \hline
\end{tabular}

Table 1: Parameter values.

The velocity $\mathbf{b}(y)$ is generated by solving the following filtration problem in the fluid part $Y^{0}$ of the unit cell $Y$

$$
\begin{cases}\nabla_{y} p-\nabla_{y}^{2} \mathbf{b}=\mathbf{e}^{i} & \text { in } Y^{0} \\ \operatorname{div}_{y} \mathbf{b}=0 & \text { in } Y^{0} \\ \mathbf{b}=0 & \text { on } \partial \Sigma^{0} \\ p, \mathbf{b} \quad \text { are } Y^{0}-\text { periodic, }, & \end{cases}
$$

where $\mathbf{e}^{i}$ is the $i$ th cartesian basis vector.

Data concerning the non-dimensional velocity and the geometry of the unit are the following:

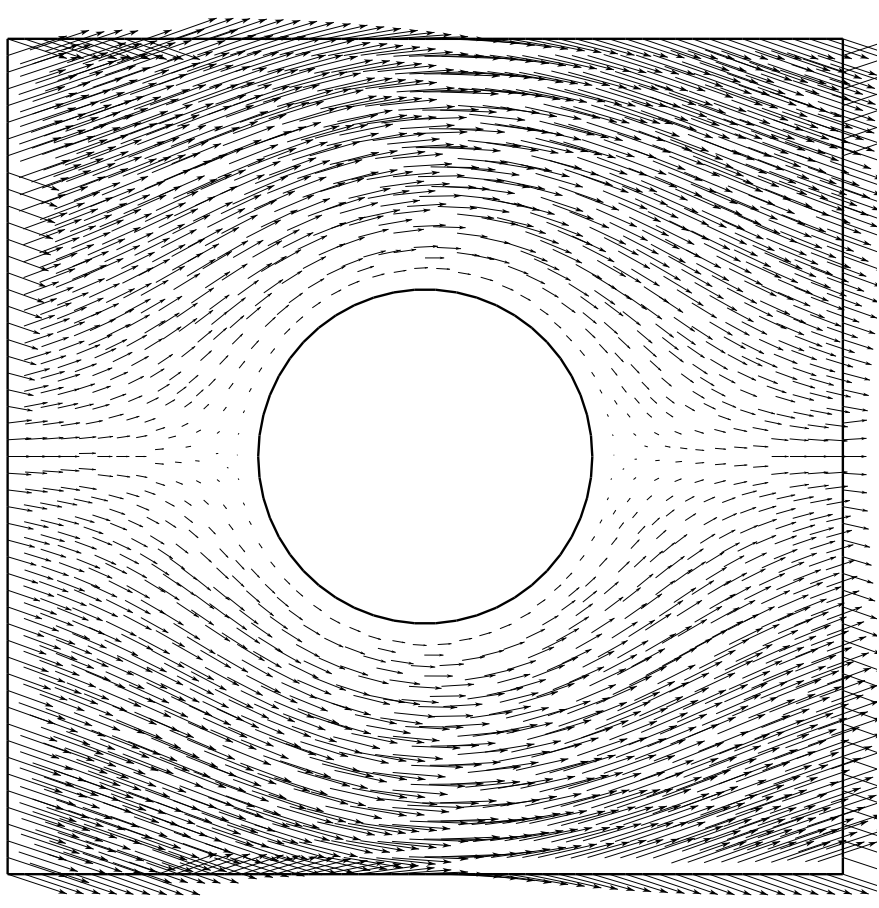

Figure 2: Fluid velocity in the unit cell.

\begin{tabular}{|l|l|}
\hline \hline PARAMETERS & VALUES \\
\hline Radius of the disk: $r$ & 0.2 \\
\hline Porosity $:\left|Y^{0}\right|=1-r^{2} \pi$ & 0.874336 \\
\hline Tortuosity $:\left|\partial \Sigma^{0}\right|_{1}=2 r \pi$ & 1.25664 \\
\hline$K_{d}$ factor: & 2.13097 \\
\hline Mean velocity $: \int_{Y^{0}} \mathbf{b}(y) d y$ & $\left(0.03853,-1.44 \cdot 10^{-5}\right)$ \\
\hline \hline
\end{tabular}

Table 2: Parameter values for the flow.

Next with given non-dimensional velocity $\mathbf{b}$ and local Péclet number $\mathbf{P e}_{l o c}$, we solve the cell problem (41). Its solution is the vectorial dimensionless concentration $\mathbf{w}=\left(w_{1}, w_{2}\right)$ displayed in Figures 3 and 4 (the grey scale goes from smaller values in white to larger values in black).

The value of the drift is then calculated using formula (44) and we get

$$
\overline{\mathbf{b}}=\left(\begin{array}{c}
0.01808 \\
-6.759 \cdot 10^{-6}
\end{array}\right)
$$

As already noted, only the symmetric part of the matrix $A^{*}$ matters for the homogenized problem (47)(48). It is naturally decomposed as $A^{*}=A_{1}+A_{2}$, where

$$
A_{1}=\left(\frac{K^{*}}{\ell K_{d}}\right)^{2}\left|\partial \Sigma^{0}\right|_{n-1} \frac{\mathrm{Pe}_{l o c}^{2}}{\mathrm{Da}_{l o c}} \int_{Y^{0}} \mathbf{b}(y) d y \otimes \int_{Y^{0}} \mathbf{b}(y) d y
$$




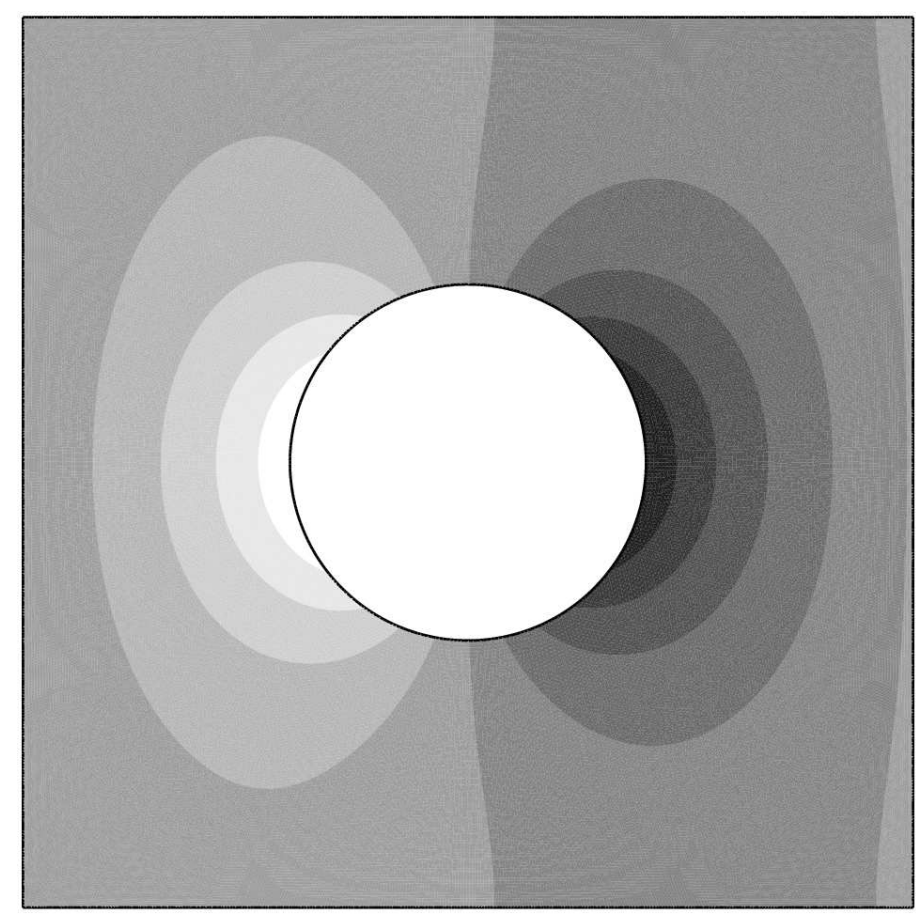

Figure 3: Dimensionless concentration $w_{1}$ for $\mathbf{P} \mathbf{e}_{l o c}=1$.

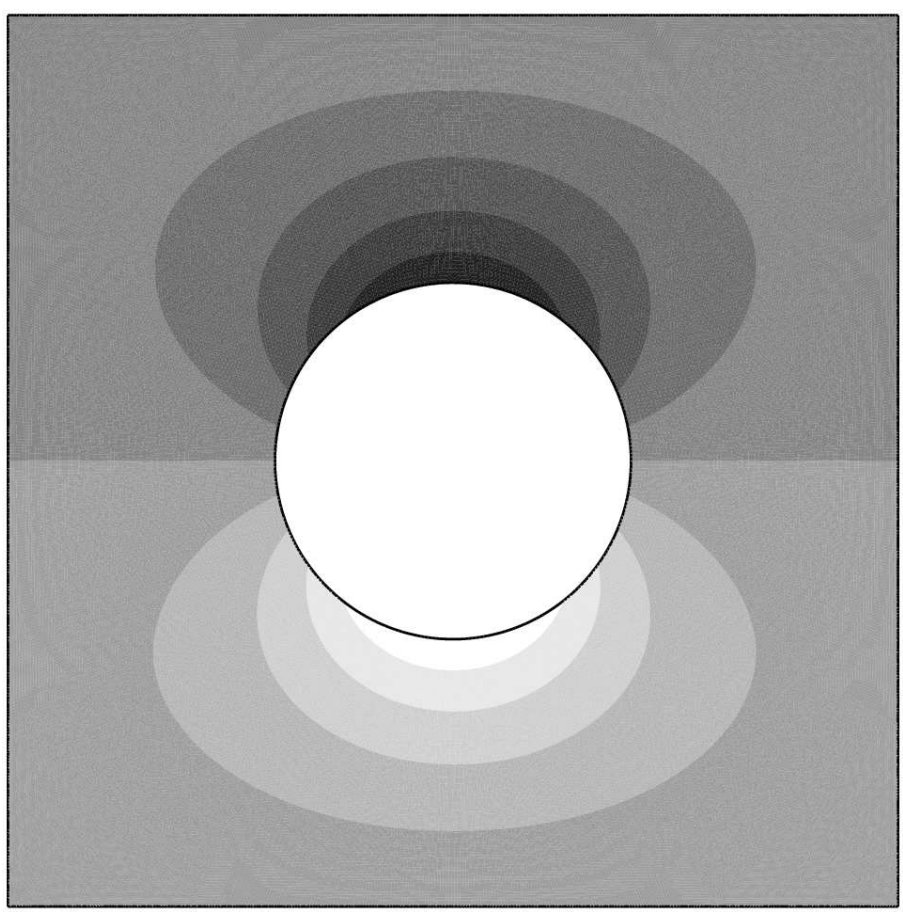

Figure 4: Dimensionless concentration $w_{2}$ for $\mathbf{P e}_{l o c}=1$.

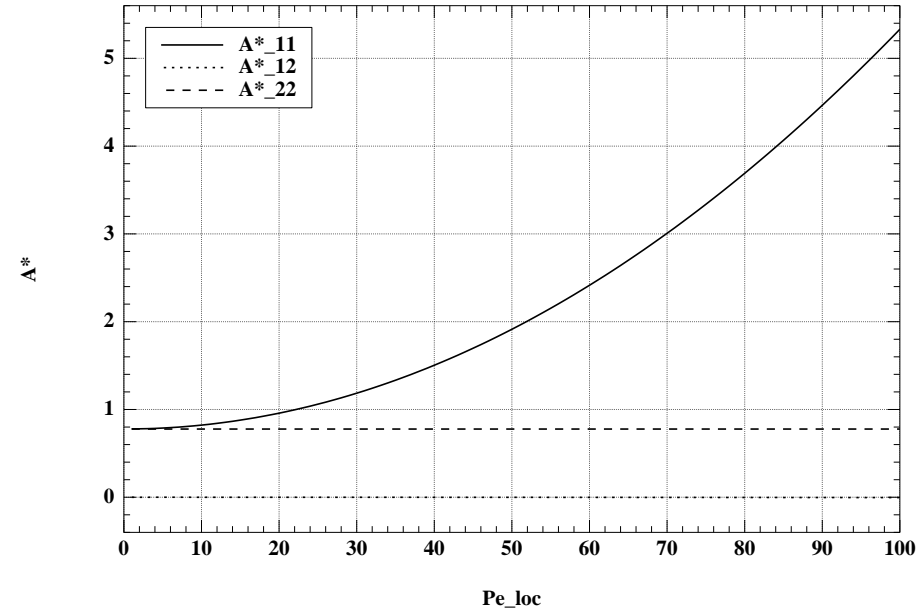

Figure 5: Entries $A_{11}^{*}, A_{12}^{*}$ and $A_{22}^{*}$ of the dispersion matrix $A^{*}$ as a function of the local Péclet's number, for Daloc $=1$

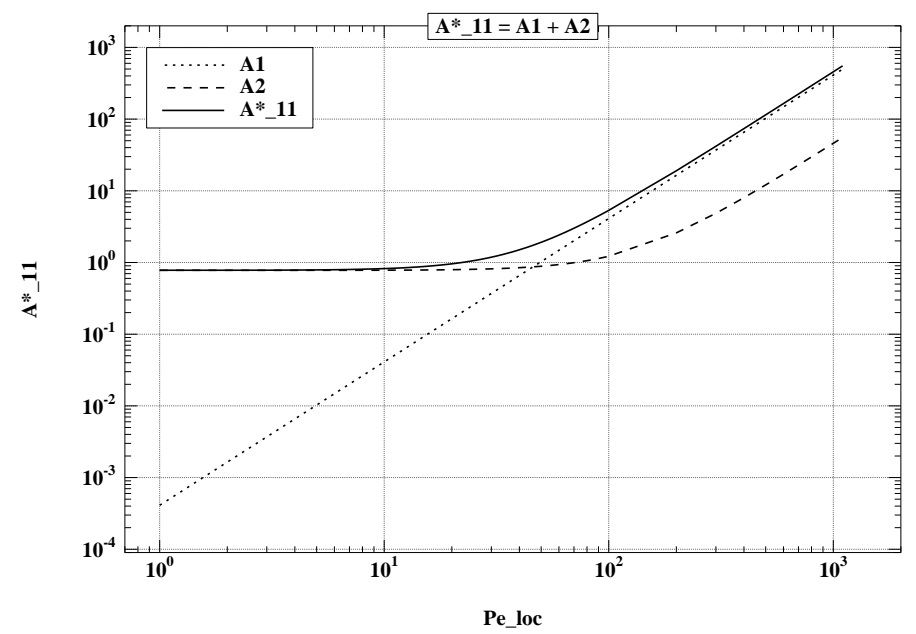

Figure 6: Log-log plot of the $(1,1)$ entry of the dispersion matrix $A^{*}=A_{1}+A_{2}$, together with its 2 components $A_{1}$ and $A_{2}$, as a function of the local Péclet's number, for $D a_{l o c}=1$

and

$$
A_{2}=\int_{Y^{0}}\left(\mathbf{I}+\nabla_{y} \mathbf{w}(y)\right)\left(\mathbf{I}+\nabla_{y} \mathbf{w}(y)\right)^{\mathcal{T}} d y .
$$

For $\mathbf{P e}_{l o c}=1$ and $\mathbf{D a} \mathbf{a}_{l o c}=1$, we get

$$
\begin{gathered}
A_{1}=\left(\begin{array}{cc}
4.10 \cdot 10^{-4} & -3.43 \cdot 10^{-7} \\
-3.43 \cdot 10^{-7} & 2.87 \cdot 10^{-11}
\end{array}\right), \\
A_{2}=\left(\begin{array}{cc}
0.778 & -1.49 \cdot 10^{-4} \\
-1.49 \cdot 10^{-4} & 0.776
\end{array}\right) \text { and } \\
A^{*}=\left(\begin{array}{cc}
0.779 & -1.50 \cdot 10^{-4} \\
-1.50 \cdot 10^{-4} & 0.776
\end{array}\right) .
\end{gathered}
$$

It is important to have $A^{*}$ as a function of $\mathbf{P} \mathbf{e}_{l o c}$ and $\mathbf{D a}_{l o c}$. It is shown on figures which follow. 


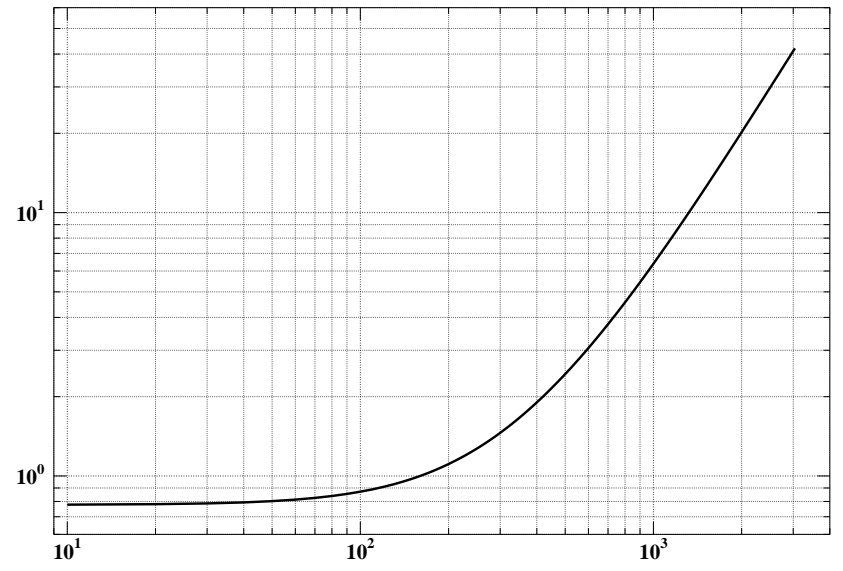

Figure 7: Log-log plot of the longitudinal dispersion $A_{11}^{*}$ as a function of the local Péclet's number, in the absence of chemical reactions, $K^{*}=0$

We do not make a direct comparison with the solution of the full physical problem because its numerical solution is costly, due to dominant Péclet and Damköhler numbers. However we compare our results to those obtained in the absence of chemical reactions (i.e., taking $K^{*}=0$ ). For large local Péclet's number the longitudinal dispersion $A_{11}^{*}$ scales like $\mathbf{P e}_{l o c}^{2}$ with chemical reactions (see the slope on Figure $6)$ while it roughly scales like $\mathbf{P e}_{l o c}^{1.7}$ without chemical reactions (see the slope on Figure 7). Our numerical results in the absence of chemical reactions are in close agreement with the corresponding ones in the literature, see e.g. [37] (Figures 7 and 8, page 2359) and [34] (Figures 18 and 20, pages 2559-2560).

\section{Conclusion}

In this article we presented a new multiple scale expansion method, which we named two-scale expansion with drift. It allowed us to determine the dispersion tensor for a reactive flow problem with dominant Péclet and Damköhler's numbers. We established that the physical concentration of solute in the fluid is approximated by $c^{e f f}(x, t)=c_{R} u_{0}\left(\frac{t}{T_{D}}, \frac{x}{L_{R}}-\right.$ $\left.\frac{t}{L_{R} K_{d}} \int_{Y^{0}} \mathbf{v}^{*}(y) d y\right)$ satisfying the problem

$$
\begin{gathered}
K_{d} \frac{\partial c^{e f f}}{\partial t}+\frac{1}{K_{d}}\left(\int_{Y^{0}} \mathbf{v}^{*}(y) d y\right) \cdot \nabla c^{e f f}- \\
\operatorname{div}\left(D^{*} A^{*} \nabla c^{e f f}\right)=0 \text { in } \mathbb{R}^{n} \times(0, T)
\end{gathered}
$$

$$
\left.c^{e f f}\right|_{t=0}=\frac{\left|Y^{0}\right| c^{0}(x)+\frac{1}{\ell}\left|\partial \Sigma_{0}\right|_{n-1} \hat{c}^{0}(x)}{\left|Y^{0}\right|+\frac{K^{*}}{\ell}\left|\partial \Sigma_{0}\right|_{n-1}} \text { in } \mathbb{R}^{n} .
$$

The derived model is much more complex than classical ones used in practical simulations (see e.g. [18]). It is validated at the mathematical level of rigor in [6], where it is proved for any finite time interval $(0, T)$, we have

$$
\int_{0}^{T} \int_{\mathbb{R}^{n}}\left|u_{\varepsilon}(t, x)-u_{0}\left(t, x-\frac{\mathbf{P} \mathbf{e}_{l o c} \overline{\mathbf{b}}}{\varepsilon} t\right)\right|^{2} d t d x \rightarrow 0,
$$

as $\varepsilon \rightarrow 0$. It is difficult to compare our model with those obtained using volume averaging as in [32]. Indeed the results of [32] are valid only for tubes and the models do not correspond. In any case our computed longitudinal dispersion $A_{11}^{*}$, in the absence of chemistry, is very similar to those computed in [37] and [34]. However, there is a strong dependence of our result on the chemistry terms and it seems to be relatively new in the literature. Of course an experimental validation of our model should be the topic of future work but it is out of the scope of the present paper.

Our homogenized model contributes to the understanding of effective reactive flows with dominant Péclet's and Damköhler's numbers. We give a relatively simple method of calculating the dispersion tensor, which can be used not only for the periodic media but also for random statistically homogeneous porous media. Expression obtained for the homogenized tensor shows that the size of dispersion could be very much increased in function of the characteristic numbers.

\section{References}

[1] G. Allaire, Homogenization and two-scale convergence, SIAM J. Math. Anal. 23, 6, pp.1482-1518 (1992).

[2] G.Allaire, One-Phase Newtonian Flow, in Homogenization and Porous Media, ed. U.Hornung, Springer, NewYork, 1997, p. 45-68.

[3] G. Allaire, Periodic homogenization and effective mass theorems for the Schrödinger equation, in Quantum transport. Modelling, analysis and asymptotics. N. Ben Abdallah and G. Frosali eds., pp.1-44, Lecture Notes in Mathematics 1946, Springer (2008).

[4] G. Allaire, A. Damlamian and U. Hornung, Two-scale convergence on periodic surfaces and applications, In Mathematical Modelling of Flow through Porous Media, Bourgeat AP, Carasso C, Luckhaus S, Mikelić A (eds). World Scientific: Singapore, 1995; p. 15-25. 
[5] G. Allaire, A.L. Raphael, Homogenization of a convectiondiffusion model with reaction in a porous medium. C. $\mathrm{R}$. Math. Acad. Sci. Paris 344 (2007), no. 8, p. 523-528.

[6] G. Allaire, A. Mikelić, A. Piatnitski, Homogenization approach to the dispersion theory for reactive transport through porous media, preprint.

[7] B. Amaziane, A. Bourgeat, M. Jurak, Effective macrodiffusion in solute transport through heterogeneous porous media. Multiscale Model. Simul. 5 (2006), no. 1, 184-204.

[8] J.L. Auriault, P.M. Adler, Taylor dispersion in porous media : Analysis by multiple scale expansions, Advances in Water Resources, Vol. 18 (1995), p. 217-226.

[9] Auriault, J. L., Lewandowska, J., Macroscopic modelling of pollutant transport in porous media, Arch. Mech. 45(I) (1993) $51-64$.

[10] A. Bensoussan, J.-L. Lions, G. Papanicolaou, Asymptotic analysis of periodic structures., North Holland, 1978.

[11] E. Canon, H. Bensmina, P. Valentin, On the Modelling of Generalized TaylorAris Dispersion in Chromatographs via Multiple Scales Expansions, Transport in Porous Media 36: $307-339,1999$.

[12] R.G. Carbonell, S. Whitaker, Dispersion in pulsed system, II-Theoretical developments for passive dispersion in porous media, Chem. Engng Sci., 38 (1983), p. 1795-1802.

[13] C. Choquet, A. Mikelić, Laplace transform approach to the rigorous upscaling of the infinite adsorption rate reactive flow under dominant Peclet number through a pore, Applicable Analysis, Vol. 87, No. 12, December 2008, 13731395.

[14] P. Donato, A. Piatnitski, Averaging of nonstationary parabolic operators with large lower order terms, GAKUTO International Series, Math. Sci. Appl., Vol. 24 (2005), Multi Scale Problems and Asymptotic Analysis, pp. 153-165.

[15] C.J. van Duijn, P. Knabner, Travelling Waves in the Transport of Reactive Solutes through Porous Media: Adsorption and Binary Ion Exchange - Part 1, Transport in Porous Media, Vol. 8 (1992), p. 167-194.

[16] C.J. van Duijn , A. Mikelić, I.S. Pop, C. Rosier, Effective Dispersion Equations For Reactive Flows With Dominant Peclet and Damkohler Numbers, In: Guy B. Marin, David West and Gregory S. Yablonsky, editors: Advances in Chemical Engineering, Vol 34, Academic Press, 2008, pp. $1-45$.

[17] A. Fannjiang, G. Papanicolaou, Convection enhanced diffusion for periodic flows, SIAM J. Appl. Math., Vol. 54 (1994), pp. 333-408.

[18] J.C.Friedly, J. Rubin, Solute transport with multiple equilibrium - controlled or kinetically controlled chemical reactions, Water Resour. Res., Vol. 28 (1992), pp. 1935-1953.

[19] V. Giovangigli, Multicomponent Flow Modeling, Birkhäuser, Boston, 1999.

[20] S. Heinze, Diffusion-advection in cellular flows with large Peclet numbers, Arch. Ration. Mech. Anal., Vol. 168 (2003), pp. 329-342.

[21] U.Hornung, W.Jäger, Diffusion, convection, adsorption, and reaction of chemicals in porous media, J. Differ. Equat. 92 (1991), 199-225.

[22] U.Hornung, W.Jäger, A.Mikelić : Reactive Transport through an Array of Cells with Semi-Permeable Membranes, Modélisation Mathématique et Analyse Numérique ( $\left.M^{2} A N\right)$, Vol. 28 (1994), p. 59-94.
[23] P. Knabner, C.J. van Duijn, S. Hengst, An analysis of crystal dissolution fronts in flows through porous media. Part 1: Compatible boundary conditions, Advances in Water Resources, Vol. 18 (1995), pp. 171-185.

[24] J. Lewandowska, J.-L. Auriault, S. Empereur, P. Royer, Solute diffusion in fractured porous media with memory effects due to adsorption, C. R. Mecanique 330 (2002) 16.

[25] E. Marušić-Paloka, A. Piatnitski, Homogenization of a nonlinear convection-diffusion equation with rapidly oscillating coefficients and strong convection, Journal of London Math. Soc., Vol. 72 (2005), No. 2, p. 391-409.

[26] R. Mauri, Dispersion, convection and reaction in porous media, Phys. Fluids A, Vol. 3 (1991), p. 743-755.

[27] C.C. Mei, Method of homogenization applied to dispersion in porous media, Transport in Porous Media, 29 (1992), 261-274.

[28] A. Mikelić, Recent Developments in Multiscale Problems Coming from Fluid Mechanics, in Trends in Nonlinear Analysis, M. Kirkilionis, S. Krömker, R. Rannacher, F. Tomi (eds.), Springer Verlag, Heidelberg, 2002, pp. 225267.

[29] A. Mikelić, V. Devigne, C.J. van Duijn, Rigorous upscaling of the reactive flow through a pore, under dominant Peclet and Damkohler numbers, SIAM J. Math. Anal., Vol. 38, Issue 4 (2006), p. 1262-1287.

[30] M. Neuss-Radu, Some extensions of two-scale convergence. C. R. Acad. Sci. Paris Sr. I Math. 322 (1996), no. 9, p. 899-904.

[31] G. C. Papanicolaou, Diffusion in random media, Surveys in applied mathematics, Vol. 1, 205-253, Plenum, New York, 1995.

[32] M.A. Paine, R.G. Carbonell, S. Whitaker, Dispersion in pulsed systems - I, Heterogeneous reaction and reversible adsorption in capillary tubes, Chemical Engineering Science, Vol. 38 (1983), p. 1781-1793.

[33] O. Pironneau, F. Hecht, A. Le Hyaric, FreeFem ++ version 2.15-1, http://www.freefem.org/fft+/.

[34] M. Quintard, S. Whitaker, Transport in ordered and disordered porous media: volume-averaged equations, closure problems, and comparison with experiment, Chem. Engng Sci., Vol. 48, (1993), no. 14, p. 2537-2564.

[35] H-K. Rhee, R. Aris, N.R. Amundson, First-Order PDEs, Vol. 1: Theory and Applications of Single Equations; Vol. 2: Theory ad Applications of Hyperbolic Systems of Quasilinear Equations, Dover, New York, 2001.

[36] J. Rubinstein, R. Mauri, Dispersion and convection in porous media, SIAM J. Appl. Math., Vol. 46 (1986), p. $1018-1023$.

[37] J. Salles, J.-F. Thovert, R. Delannay, L. Prevors, J.-L. Auriault, P. M. Adler, Taylor dispersion in porous media. Determination of the dispersion tensor, Phys. Fluids A 5 (10), October 1993, p. 2348-2376.

[38] E. Sanchez-Palencia, Non-Homogeneous Media and Vibration Theory, Springer Lecture Notes in Physics 127, Springer-Verlag, Berlin, 1980.

[39] G.I. Taylor, Dispersion of soluble matter in solvent flowing slowly through a tube, Proc. Royal Soc. A, Vol. 219 (1953), p. 186-203. 